\title{
To investigate the Temporal and Spatial correlation behavioral characteristics of MIMO fading channels
}

Rachna Mahey, Jyoteesh Malhotra

ECE Department, Guru Nanak Dev University, Regional campus, Jalandhar, INDIA rachnamaheyr@gmail.com

ECE Department, Guru Nanak Dev University, Regional campus, Jalandhar, INDIA

\section{ABSTRACT}

jyoteesh@gmail.com

This paper deals with the analysis of performance bounds of narrowband multiple-input multiple-output (MIMO) fading channels considering the transmission links from the mobile station (MS) to the base station (BS) through non-line-of-sight (NLOS) propagation conditions. It is assumed that an infinite number of local scatterers surround the MS and the BS. The Temporal as well as Spatial correlation properties of channel models are explored along with their capacity. The effect of number of scatterers; Doppler frequency; and isotropic/non-isotropic scattering conditions, on MIMO channel models is investigated in terms of statistical properties of one ring and two ring models, i.e. Time Autocorrelation Function (ACF) and two-dimensional (2D) Space Cross-correlation Function (CCF).

\section{Keywords}

Mimo fading channels, Deterministic channel modeling, Time ACF, Space CCF, Capacity

\section{Academic Discipline And Sub-Disciplines}

Electronics and Communications Engineering, Specialization in Communication

\section{SUBJECT CLASSIFICATION}

Wireless Communications, MIMO Fading Channels

\section{TYPE (METHOD/APPROACH)}

Analysis

\section{Council for Innovative Research}

Peer Review Research Publishing System

\section{Journal: INTERNATIONAL JOURNAL OF COMPUTERS \& TECHNOLOGY}

Vol.14, No.7

www.ijctonline.com, editorijctonline@gmail.com 


\section{INTRODUCTION}

The propagation conditions, determine the channel characteristics, and the channel capacity of a multiple-input multipleoutput (MIMO) system, thus it is important to develop MIMO channel models, to investigate the impact of the channel parameters on the capacity of MIMO systems in various propagation environments [1]. The appropriate MIMO fading channel models can help in reducing the costs of research and development, by simplifying the required computations and measurements on the design under different scenarios; and hence the system performances can be predicted accurately. Therefore, the realistic models of mobile fading channel are of great importance for designing, analysis, optimization, and testing of wireless communication systems [2]. A sophisticated MIMO channel models are required [3] to develop advanced mobile communication systems employing multiple antennas at the transmitter (MT) and receiver side (MR), that precisely reflect all the relevant features of physical channel. For designing and testing of MIMO Mobile-to-Mobile (M2M) systems, the Geometrically-Based Stochastic Model (GBSM) [4] is applied for narrowband MIMO channels. The most well-known narrowband MIMO channel models are; the one-ring model and the two-ring model. In M2M communication channels [5], the transmitter and receiver both are in motion; means one vehicle moving in a city tries to communicate with other moving vehicle at other location; for example police cars, emergency vehicles etc. Thus, the Lineof-Sight (LOS) is obstructed by the buildings or any other type of obstacle (may be scatterers) between the transmitter and receiver. The One-Ring Model assumes that the BS (transmitter) is not obstructed by any local scatterers, while the MS (receiver) is located in the center of a ring of surrounding scatterers [6]. This sort of assumption is suitable for propagation environments in the rural and sub-urban areas, where tall BS antennas are used. The Two-Ring Model assumes that both the transmitter and receiver are surrounded by scatterers [6], as in indoor channels.

In the literature, several models exist that allow for an appropriate simulation of mobile channels. Oestges, C. and Clerckx, B. [4], illustrate that an important aspect of MIMO channel modeling consists of describing the correlations between pairs of transmitter and receiver antennas. For Geometry-based models [7], instead of modeling the environment precisely, the scatterers having complex Gaussian properties are specified by a spatial distribution. Parizi, F. Z. and Mehrjoo, M. [8] presents a MIMO geometry-based stochastic channel models (GSCM) for regular shaped scattering environments, such as, one-ring and two-ring models. Avazov, N. and Patzold, M. [9], investigates the geometrical one-ring scattering model assuming single-bounce scattering in LOS and NLOS propagation environments. Analytical solutions are analyzed for the space-time-frequency CCF (STF-CCF), the 2D space CCF, the time-frequency CCF (TF-CCF), the temporal ACF, and the frequency correlation function (FCF). The comparison results [8], [9] reveal an excellent agreement between theory and measurements, which confirms that the derived reference model is valid. Wang, S. and Abdi, A. [10], also proposes a single-bounce two-ring statistical model for time-varying MIMO flat fading channels, that provides a useful channel characterization required for efficient design and performance prediction of multi-antenna systems. Talha, B. and Patzold, M. [11], derive a stochastic narrowband MIMO reference channel model from the geometrical three-ring scattering model. The general analytical solutions are found for the four-dimensional (4D) space-time CCF, 3D spatial CCF and the temporal ACF, which show that various CCFs describing the simulation model closely approximate the corresponding CCFs of the reference model. Cheng, X. and Laurenson, D. I. [12], extends the narrowband one-ring MIMO model to a new wideband multi-ring model for macro-cell scenarios. Patzold, M. [13] shows that the parameters of the simulation model can be determined by using the EMEDS (Extended Method of Exact Doppler Spread) and LPNM (Lp-norm method). The application of the former method is highly recommended for the isotropic scattering scenarios, while the latter exhibits its full capabilities when the $A O D$ and $A O A$ are non-uniformly distributed, as it is generally the case in real-world environments (non-isotropic scattering).

This paper contributes to the one ring and two-ring models, by focusing on their performance analysis under different channel conditions. Stochastic simulation models for MIMO channels under the assumption of isotropic and non-isotropic scattering are investigated. The starting point of the performance evaluation is a non-realizable stochastic MIMO reference model as it has been developed for the one ring and two-ring scattering models in [6] [11]. General analytical solutions are provided for the 2D space CCF, time autocorrelation function (ACF), and Capacity distribution [3]. To compute the parameters of the simulation model, two methods are proposed [6] [14]. The first method is an extension of the MEDS, i.e. extended method of exact Doppler spread (EMEDS), which provides the closed-form expressions for the model parameters. EMEDS is developed for propagation scenarios described by uniformly distributed angles of departures (AOD) and angles of arrivals (AOA). The second method is Lp-norm method (LPNM) which provides the model parameters for many types of distribution functions of AOD and AOA; as the Von-Mises distribution [2] and the Gaussian distribution, however LPNM is more complex. In this paper, the Temporal and Spatial correlation behavioral characteristics of MIMO fading channels are analyzed and the effect of number of scatterers; Doppler frequency; and Isotropic/Nonisotropic scattering conditions, on MIMO channel models is investigated. The proposed methods can provide an important framework for system designers of advanced mobile communications, to verify new transmission concepts employing MIMO techniques under realistic propagation conditions. The rest of this paper is organized as: The geometrical, stochastic reference, stochastic simulation and deterministic simulation models for geometrical one-ring and two ring models, is described briefly in Section 2. The simulation methodology and environment is illustrated in Section 3 . The performance results are evaluated in Section 4.

\section{DETERMINISTIC CHANNEL MODELING}

The simulation models are based on the principle of Deterministic channel modeling [6] [13]. Starting from the geometrical one-ring (or two ring) model, a stochastic and a deterministic simulation model is derived via a non-realizable stochastic reference model. The generalized concept of deterministic channel modeling used in this section is described by Figure 1 : 


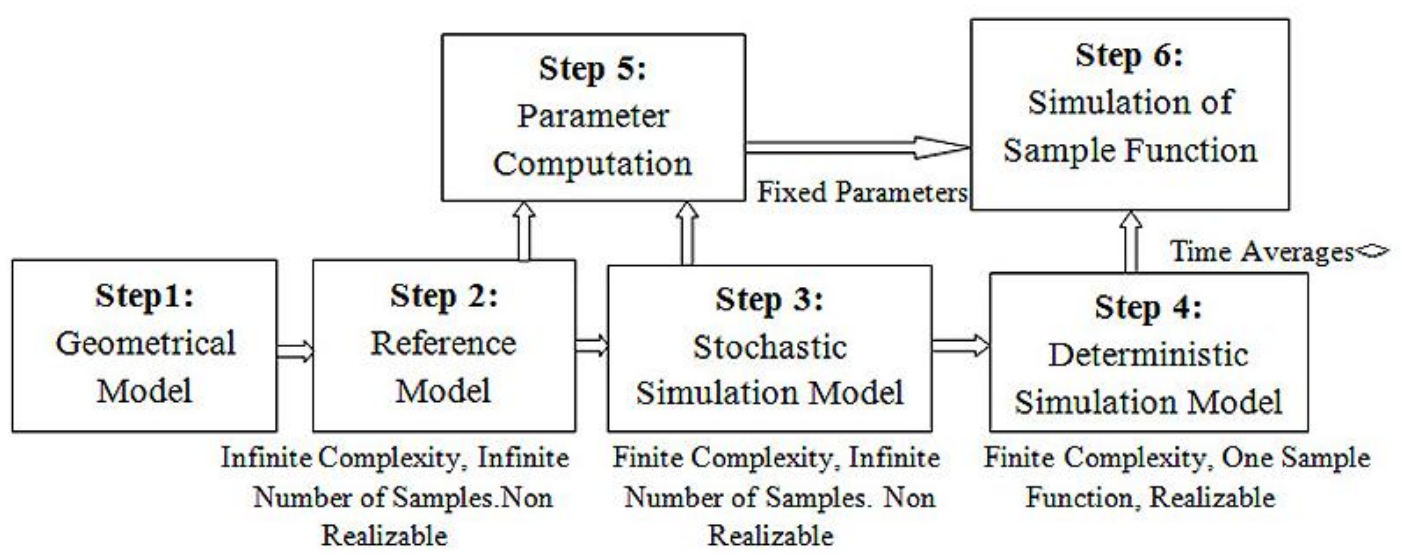

Figure 1: The steps of deterministic channel modeling [13]

\subsection{The One-Ring MIMO Channel Model:}

The One-ring model is preferred in modeling sub-urban environments [3], where the BS and MS are considerably distant from each other and the BS is at much higher height than the MS. Thus, it is assumed that the BS (transmitter) has little or no scatterers surrounding it, as may be placed on a very high point, for e.g. a hill, mountain or Tower; while the MS (receiver) is placed at a much lower point and is assumed to be surrounded by many scatterers existing on a ring around the MS [8]. In Mobile-to-Mobile (M2M) communication systems, an example of this model may be an airplane or helicopter communicating with a ground vehicle. In this subsection, modeling, analysis, and simulation of one ring model are described briefly.

\subsubsection{Geometrical One Ring Scattering Model:}

The geometrical one ring model for MIMO channel employs multi-element antenna arrays. The Figure 2 contains description of all components of the model with local scatterers around the MS. It is assumed that the LOS path between the $\mathrm{BS}$ and the MS is obstructed. Here, $D$ is the distance between MS and $\mathrm{BS}$; $R$ is radius of the ring of scatterers, $a_{\text {max }}^{T}$ denotes the maximum AOD (angle of departure) observed from the BS; $a_{n}{ }^{R}$ is the AOA (angle of arrival) at MS; $\beta_{T}$ is the BS's tilt angle (in degree); $\beta_{R}$ is the MS's tilt angle; $d_{T}$ is antenna inter-element spacing at $B S ; d_{R}$ is antenna element spacing at mobile station; $a_{\text {max }}^{T}$ is one half of the maximum AOD observed at the BS; and $a_{v}$ is the MS's angle of motion [3] [6].
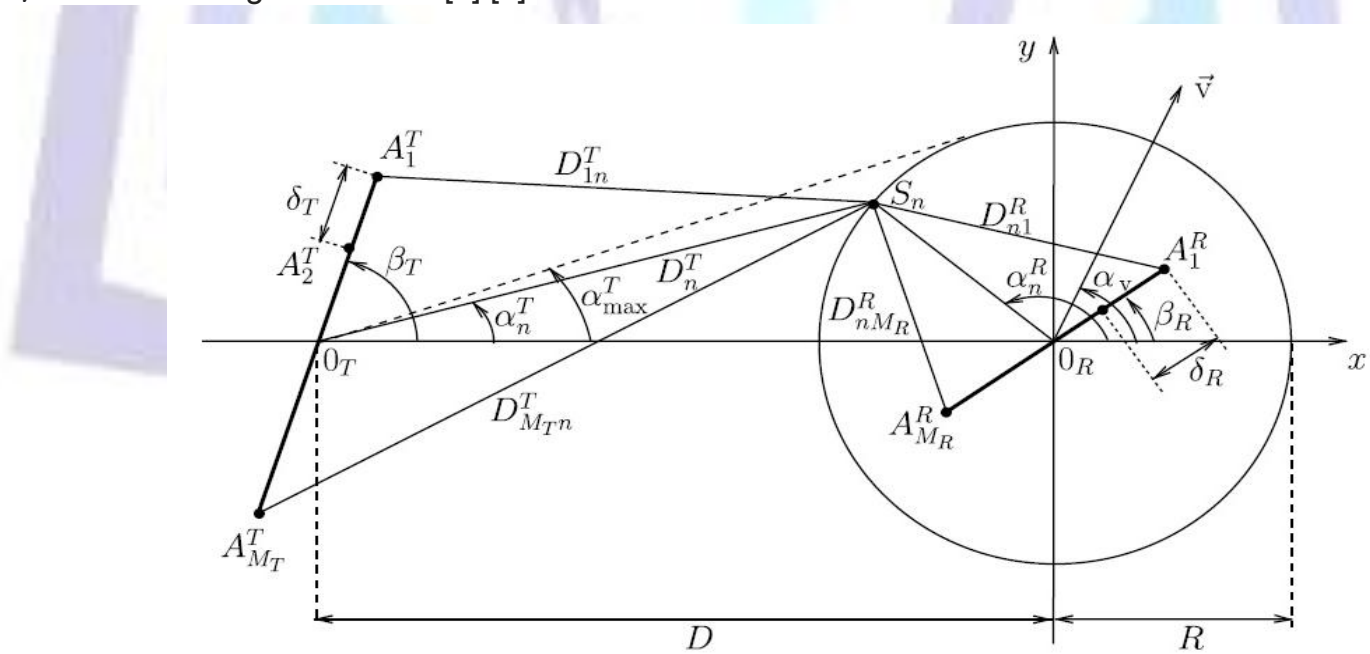

Figure 2: Geometrical model (one-ring model) for an $M_{T} \times M_{R}$ MIMO channel with local scatterers around MS [8]

\subsubsection{Reference Model for the One-Ring MIMO Channel Model:}

For One-ring channel model, $M_{T} \times M_{R}$ MIMO channel with local scatters lying on a ring around the MS is assumed as a reference model. It is assumed that the number of local scatterers is infinite, i.e. $(\mathrm{N}=\infty)$. The complex channel gain $h_{k l}(t)$ of the reference model describing the link from the th transmitter antenna element $A^{T} I\left(I=1,2, \ldots, M_{T}\right)$ to the $k^{\text {th }}$ receiver antenna element $A^{R} k\left(k=1,2, \ldots, M_{R}\right)$ is expressed as [13]:

$$
h_{k l}(t)=\lim N \rightarrow \infty \quad \frac{2}{\sqrt[N]{N}} \sum_{n=1}^{N} g k l n e^{j\left(2 \pi f n t+\theta n+\theta_{0}\right)}
$$


Here $\theta n$ is the phase change due to the interaction with the $n$th scatterer $S n$. The time ACF of the complex channel gain $h_{k l}(t)$ derived from the space-time CCF is obtained as [6]:

$$
\begin{aligned}
& r h_{k l}(T):=E\left\{h_{k l}(t) h^{*}{ }_{k l}(t+T)\right\} \\
& =\int_{-\mathbb{\pi}}^{\mathbb{m}} e^{-j 2 \pi f \max \cos (\alpha R-\alpha v) \tau} p_{\alpha R}\left(\alpha^{R}\right) d \alpha^{R}
\end{aligned}
$$

Here * denotes the complex conjugate operator. The 2D space CCF is given as [13]:

$$
\begin{aligned}
& \rho\left(\delta_{T}, \delta_{R}\right):=E\left\{h_{11}(t) h_{22}^{*}(t)\right\} \\
& \quad \int_{-\pi}^{\mu} a^{2}\left(\delta_{T,}, \alpha^{R}\right) b^{2}\left(\delta_{R,}, \alpha^{R)} p_{\alpha R}\left(\alpha^{R}\right) d \alpha^{R}\right.
\end{aligned}
$$

Also, the instantaneous channel capacity of one ring channel model $\mathrm{C}(t)$ is defined as [13]:

$$
\mathrm{C}(\mathrm{t})=\log _{2}\left[\operatorname{det}\left(\mathrm{I}_{2}+\frac{\mathrm{P} \text { Total }}{2 \text { No }} \mathrm{H}(\mathrm{t}) \mathrm{H}^{\mathrm{H}}(\mathrm{t})\right)\right] \quad[\mathrm{bits} / \mathrm{s} / \mathrm{Hz}]
$$

\subsubsection{Simulation Models for the One-Ring MIMO Channel Model:}

An efficient simulation model is obtained from given reference model derived from the plane wave model by applying the generalized concept of deterministic channel modeling. In the third step, a stochastic simulation model is derived from the reference model simply by replacing the infinite number of scatterers by a finite number $N$. The fourth step is the step from the stochastic simulation model to the corresponding deterministic simulation model, which is performed by fixing all model parameters, including the phases.

Stochastic Simulation Model: The time ACF $\hat{r}^{\hat{h}} h_{k l}(\tau)$ of the complex channel gain $\hat{h}_{k l}(t)$ of the stochastic simulation model is expressed as [6]:

$$
\begin{aligned}
\hat{r} h_{k l}(T):=E & \left.\left\{\hat{h}_{k l}(t) \hat{h}^{*} k l(t+T)\right\}\right|_{\theta n} \\
& =\frac{1}{N} \sum_{n=1}^{N} e^{-j 2 \pi f n T}
\end{aligned}
$$

Finally, the 2D space CCF $\rho^{\wedge}(\delta T, \delta R)$ of the stochastic simulation model is derived as follows [6]:

$$
\begin{aligned}
\rho^{\wedge}(\delta T, \delta R) & :=\left.E\left\{\hat{h}_{11}(t) \hat{h}^{*}{ }_{22}(t)\right\}\right|_{\theta n} \\
& =\frac{1}{N} \Sigma_{n=1}^{N} e^{2 \pi}\left\{\frac{\partial T}{\lambda_{0}} \cos (\beta T)+\alpha T \max \sin (\beta T) \sin (\alpha R n)++\frac{\partial R}{\lambda_{0}} \cos (\alpha R n-\beta R)\right\}
\end{aligned}
$$

Deterministic Simulation Model: The practical realization of a stochastic simulation model requires theoretically the simulation of an infinite number of sample functions. The time ACF of the deterministic process $h^{\sim} k l(t)$ is obtained as [6]:

$$
\tilde{r} h_{k l}(T)=r h_{k l}(T)
$$

The $2 \mathrm{D}$ space CCF of the deterministic simulation system, which is derived as follows [6]:

\subsubsection{Parameter Computation Methods (isotropic and non-isotropic):}

$$
\begin{aligned}
& \rho^{\sim}\left(\delta_{T}, \delta_{R}\right):=\rho^{\wedge}\left(\delta_{T}, \delta_{R}\right) \\
& \text { opic and non-isotropic): }
\end{aligned}
$$

The objective of any parameter computation method is to find proper values for model parameters, such that the statistical properties of the deterministic (stochastic) simulation model are sufficiently close to those of the stochastic reference model. The model parameters to be determined are the discrete AOAs $\alpha^{R} n(n=1,2, \ldots, M)$.

Extended method of exact Doppler spread (EMEDS): Isotropic scattering around the transmitter is characterized by a uniform distribution of the AOD $\alpha^{T}$. For isotropic scattering around the receiver, we employ the EMEDS to determine the set of discrete AOAs $\left\{\alpha^{R} n\right\} N n=1$. This method is a special case of the generalized method of exact Doppler spread (GMEDSq). According to the EMEDS, the model parameters $\alpha^{R} n$ are determined by [6]:

$$
\alpha^{R}{ }_{n=\frac{2 \pi}{N}}\left(n-\frac{1}{2}\right)+\alpha^{R} 0, \quad n=1,2, \ldots, N
$$

Where, $\alpha^{R} 0$ is called the angle-of-rotation.

Lp-norm method (LPNM): To describe non-isotropic scattering scenarios, it is assumed that the AOD $\alpha_{T}$ follows the von Mises distribution, for describing the measured data [2]. For non-isotropic scattering around the receiver, the LPNM can be applied. The application of this method to the present problem requires the minimization of the two $L p$-norms [14]:

$$
\begin{gathered}
E(p) 1:=\left\{\frac{1}{\tau \max } \int_{0}^{\tau \max } \| r_{h 11}(\tau)-\left.\tilde{r}_{h 11}(T)\right|^{p} d T\right\}^{1 / p} \\
E_{2}^{(p)}:=\left\{\frac{1}{\tau T \max } \frac{1}{\tau \operatorname{mmax}} \int_{0}^{\tau T \max } \int_{0}^{\tau R \max }\left|\rho\left(\delta_{T}, \delta_{R}\right)-\rho^{\sim}\left(\delta_{T}, \delta_{R}\right)\right|^{p} d \delta_{T} d \delta_{R}\right\}^{1 / p}
\end{gathered}
$$




\subsection{Two-Ring MIMO Channel Model}

The Two-ring model is similar to the One-ring model; the difference is in the vertical position of the BS, which is not as higher as that in One-ring model. So, it is assumed that the BS is also surrounded by the scatterers existing on a ring along with the MS. Therefore, the model has two rings, one surrounding the BS (which is the transmitter); and the other surrounding the MS (which is the receiver). In Mobile-to-Mobile communications, the two ring model may be seen as a helicopter flying low in the city communicating with a ground vehicle.

\subsubsection{Geometrical Two-Ring Scattering Model:}

In this subsection, the geometrical two-ring scattering model for a narrowband MIMO channel is described briefly as illustrated in Figure 3. In the two-ring model, only local scattering is considered, because it is assumed that the contributions of remote scatterers to the total received power can be neglected due to high path loss. For simplicity, it is assumed that both transmitter and receiver are equipped with only two Omni-directional antennas.

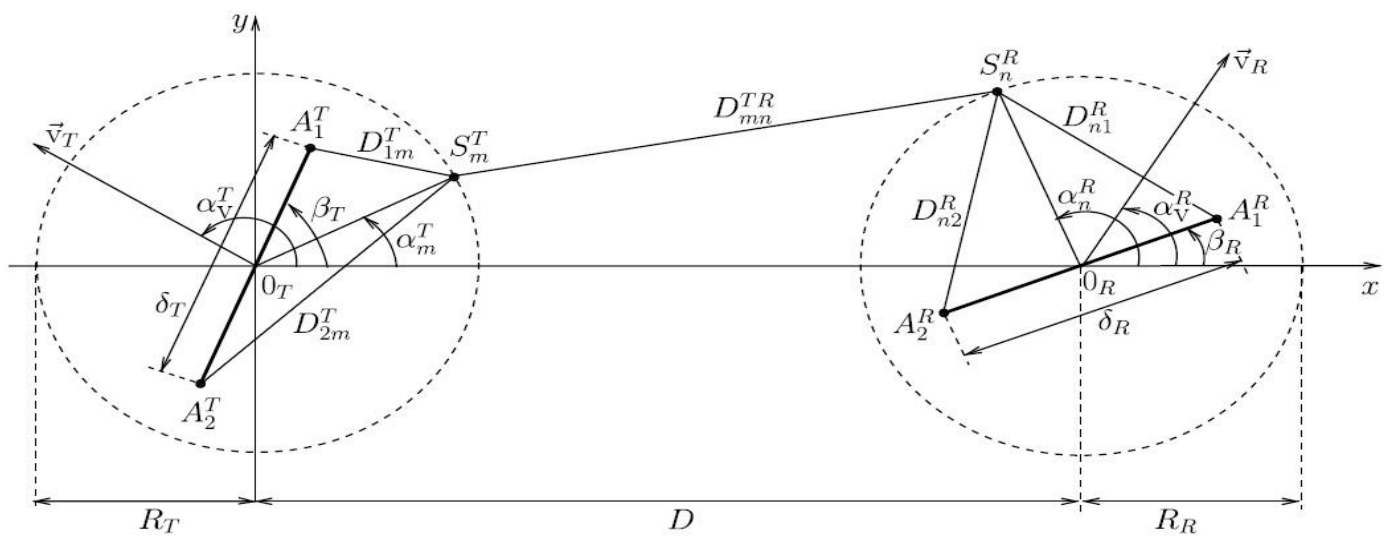

Figure 3: The geometrical two-ring model for a $2 \times 2$ MIMO channel with local scatterers around a mobile transmitter (left) and a mobile receiver (right) [8]

\subsubsection{Reference Model for the Two-Ring MIMO Channel Model:}

For two-ring reference model, the number of scatters around the receiver and transmitter is assumed to be infinite. The capacity that characterizes the channel is designated as [6]:

$$
\mathrm{C}(\mathrm{t})=\square \log \left[\operatorname{det}\left(\mathrm{I}_{2} \frac{P T}{2 N_{0}} \mathrm{H}(\mathrm{t}) \mathrm{H}^{\mathrm{H}}(\mathrm{t})\right]\right.
$$

Where, $\mathrm{I}_{2}$ is the identity matrix with two rows and two columns, $\mathrm{P}_{\mathrm{T}}$ is the total transmitted power allocated uniformly to the two antenna elements of the transmitter and $\mathrm{N}_{0}$ is the noise power. The time ACF $r^{h} k(T)$ of the complex channel gain $h_{k l}(t)$ of the transmission link from $A_{T I}$ to $A_{r k}(k, I=1,2)$ is defined by [6]:

$$
r_{h_{k l}}(T)=\rho_{T}(0, T) \cdot \rho_{R}(0, T)
$$

The 2D space CCF $\rho(\delta T, \delta R)$, defined as $\rho(\delta T, \delta R)=E\left\{h_{11}(t) h_{* 22}(t)\right\}$, equals the three dimensional space-time CCF $\rho 11,22(\delta T, \delta R, T)$ at $T=0,[6]$ i.e.,

$$
\begin{aligned}
\rho\left(\delta_{T}, \delta_{R}\right)= & \rho 11,22\left(\delta_{T}, \delta_{R}, 0\right) \\
& =\rho T\left(\delta_{T}, 0\right) \cdot \rho R\left(\delta_{R}, 0\right)
\end{aligned}
$$

\subsubsection{Simulation Models for the Two-Ring MIMO Channel Model:}

This subsubsection presents a stochastic as well as a deterministic simulation model by applying the generalized principle of deterministic channel modeling.

The Stochastic Simulation Model: From the complex channel gain $h_{11}(t)$ of the reference model, a stochastic simulation model is obtained. The $2 \mathrm{D}$ space CCF $\rho^{\wedge}\left(\delta_{T}, \delta_{R}\right)$ is expressed as [6]:

$$
\begin{aligned}
& \rho^{\wedge}\left(\delta_{T}, \delta_{R}\right)=\rho^{\wedge} 11,22\left(\delta_{T}, \delta_{R}, 0\right) \\
& =\hat{\rho}_{T}\left(\delta_{T}, 0\right) \cdot \hat{\rho_{R}}\left(\delta_{R}, 0\right)
\end{aligned}
$$

Furthermore, the time ACF $\hat{r} h_{k l}(T)$ of $\hat{h}_{k l}(t)$ is expressed in closed form as [6]:

$$
\hat{r} h_{k l}(T)=\hat{\rho} T(0, T) \cdot \hat{\rho}_{R}(0, T) \quad \forall k, l \in\{1,2\} .
$$


The Deterministic Simulation Model: The deterministic simulation model is obtained from the stochastic one by keeping all model parameters fixed, including the phases $\theta_{m n}$. The 2D space $\operatorname{CCF} \rho^{\sim}(\delta T, \delta R)$ of the deterministic MIMO channel simulator is expressed as [6]:

$$
\tilde{\rho}\left(\delta_{T}, \delta_{R}\right)=\rho T\left(\delta_{T}, 0\right) \cdot \rho_{R}\left(\delta_{R}, 0\right)
$$

Similarly, the time ACF $\tilde{r}_{k l}^{h}(T)$ of the deterministic process $\tilde{h}_{k l}(t)$ is obtained as [6]:

$$
\tilde{r}_{k l}^{h}(T)=\tilde{\rho}_{T}(0, T) \cdot \tilde{\rho}_{R}(0, T) \quad \forall k, l \in\{1,2\}
$$

\subsubsection{Parameter Computation Methods:}

In this section, two methods for the computation of the parameters determining the statistics of the MIMO channel simulation model are represented. The first method is the EMEDS [9], which is highly recommended in case of isotropic scattering. The other methods is LPNM [11] which can be applied to any given distribution of the AOD (AOA), including the von Mises distribution [2], the Gaussian distribution, the truncated Laplacian distribution, and the truncated uniform distribution [6].

\section{SIMULATION METHODOLOGY AND ENVIRONMENT}

In this paper, the simulation models are based on the principle of Deterministic channel modeling which consists of the steps shown in Figure 1. The simulation of the one ring and two ring MIMO channel models is divided into two parts. First part deals with the computation of the AODs at the transmit side, and the other part determines the AOAs where each one is computed individually. By combining these two results the channel gains as well as the impulse response which characterizes the behavior of channel model are determined. The different steps for the simulations are:

1. The AOD and AOA are computed using two fundamental methods, by simply choosing the right parameters. The autocorrelation function of the simulation model is taken as criteria to evaluate the performance of each computation method.

2. In the second step, the channel gains are computed by using the obtained parameters for multiple uncorrelated fading waveforms.

3. The option PLOT is used to sketch the performance criteria, such as temporal ACF and space-time CCF.

4. The capacity of simulated channel model is presented using ecdf (empirical cumulative distribution function).

The resulting MIMO channel simulations takes not only the spatial correlation properties at the transmit side and the receive side into account, but models also accurately the Doppler Effect. The different parameter computation methods used are EMEDS and LPNM method. For each parameter computation method, the time ACF and 2D space CCF of the simulation models are depicted as proper statistical quantities. The simulations are based on the isotropic scattering assumption, i.e., $p(\varphi T)=p(\varphi R)=1 /(2 \pi)$ and non-isotropic assumption. The tilt angles are defined as $\alpha^{\top}=\alpha^{R}=\pi / 2$, and the angle of motion $\alpha_{v}$ is set to $\pi$. The ring radii $R_{T}$ and $R_{R}$ were equal to $10 \mathrm{~m}$. A maximum Doppler frequency of $f_{\max }=91$ $\mathrm{Hz}$ and $200 \mathrm{~Hz}$ is assumed, and the wave length is set to $\lambda=0.15 \mathrm{~m}$. The parameters $\varphi(\mathrm{m}) \mathrm{T}$ and $\varphi(\mathrm{n}) \mathrm{R}$ of the stochastic simulation model have been computed by using the proposed extended MEDS with $M=20$ and $N=40$. The parameters used are as shown in Table 1.

\begin{tabular}{|c|c|c|c|}
\hline & Parameter & Isotropic Scattering & Non-Isotropic Scattering \\
\hline $\mathrm{N}$ & $\begin{array}{l}\text { Number of scatterers placed on the ring } \\
\text { centered on the receiver }\end{array}$ & 20,40 & 20,40 \\
\hline$\beta_{\mathrm{BS}}$ & Base station antenna tilt angle in degree & 90 & 90 \\
\hline$\beta_{M S}$ & Mobile station antenna tilt angle in degree & 90 & 90 \\
\hline$\alpha_{\max }^{\prime}$ & One half of the maximum AOD in degree & 2 & 2 \\
\hline $\mathrm{f}_{\max }$ & Maximum Doppler frequency & 91,200 & 91,200 \\
\hline K ( Kappa) & Spread factor of the von Mises distribution & 0 & 10.40 \\
\hline$m_{\alpha}$ & Mean AOA of the von Mises distribution & 0 & 0 \\
\hline$\alpha_{v}$ & Angle-of-motion in degree & 180 & 180 \\
\hline $\mathrm{P}$ & Parameter controlling the LPNM & 2 & 2,100 \\
\hline METHOD & Parameter computation method & $\begin{array}{l}\text { EMED (Extended MEDS } \\
\text { for is otropic scattering) }\end{array}$ & $\begin{array}{l}\text { LPNM (Lp-norm method for } \\
\text { non-isotropic scattering) }\end{array}$ \\
\hline
\end{tabular}

Table 1: Parameters used for the Simulations 


\section{RESULTS AND DISCUSSIONS}

The time ACF and 2D space CCF are considered to compute the performance bounds of MIMO channel models by varying the number of scatterers; Doppler frequency; and isotropic/non-isotropic scattering conditions. The simulation results for different parameters values coming from the EMEDS method and the LPNM method (for isotopic and nonisotropic scattering respectively) are illustrated as:

The Isotropic Scattering is investigated by choosing $\kappa=0$ and $m_{\alpha}=0$ and the method used for computing simulation parameters is EMED. Figure 4(a) and 4(b) shows the time ACF and 2D space CCF $\rho^{\wedge}{ }_{T}\left(\delta_{T}, \delta_{R}\right)$ for one ring channel model under isotropic scattering conditions. For the simulation model, an appropriate number of discrete scatterers $\mathrm{N}$ located on the rings around the source $M S$ is selected $(N=20)$. The simulation parameters chosen are; $N=20, \beta_{B S}=90, \beta_{M S}=90$, $\alpha_{\text {max }}^{\top}=2, f_{\max }=91 \mathrm{~Hz}, k=0, m_{\alpha}=0, \alpha_{v}=180$ and $p=2$. The time ACF is obtained for reference and simulation model,

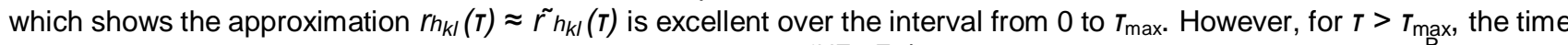
ACF's are inconsistent. The space CCF of simulation model, $\rho^{\sim}\left(\delta_{T}, \delta_{R}\right)$ is evaluated using the discrete AOAs $\alpha_{n}^{R}$ obtained by applying the EMED.

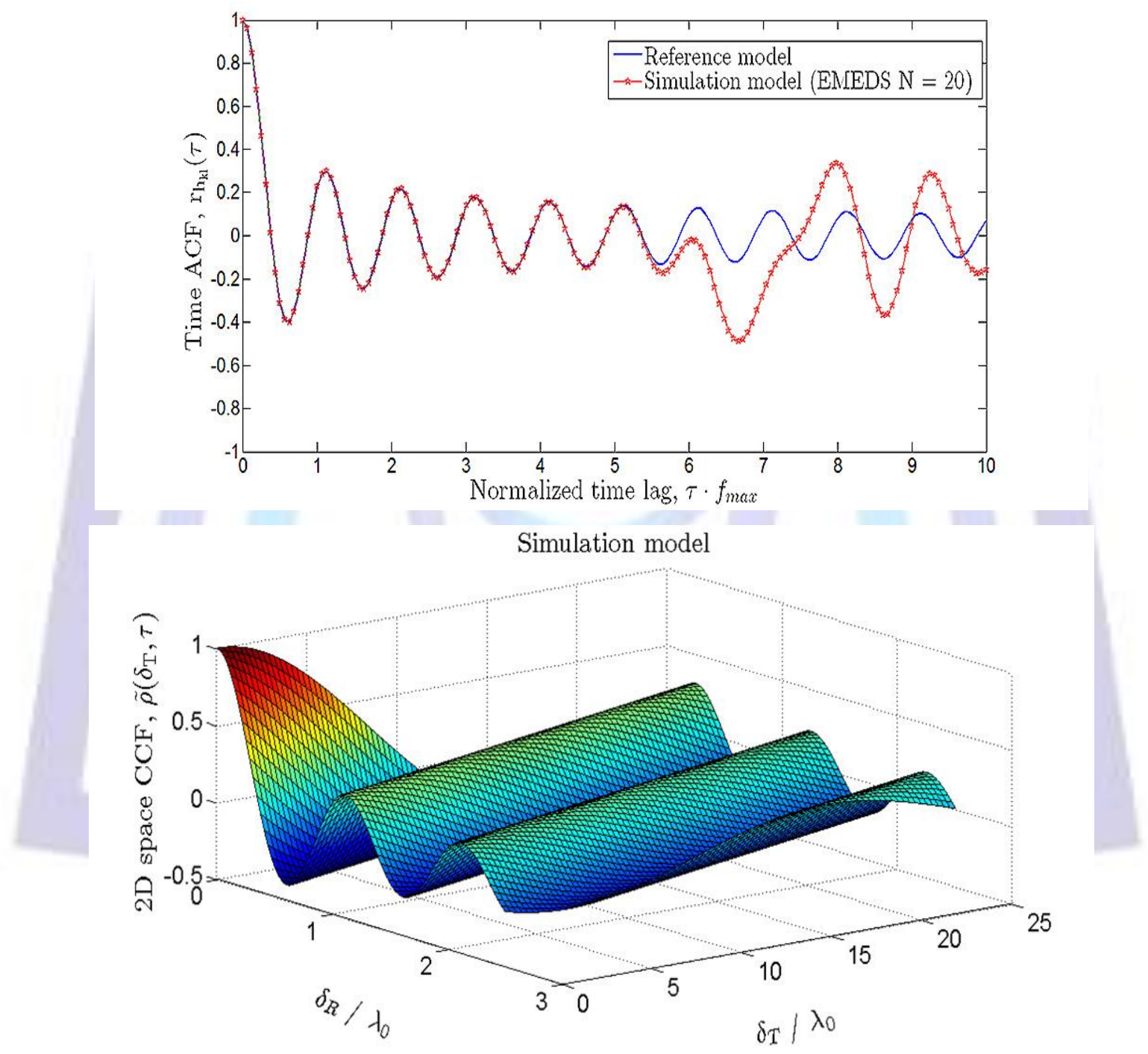

Figure 4: (a) Time ACFs of reference model and simulation model for isotropic scattering $\left(E M E D, N=20, f_{\max }=\right.$ $91 \mathrm{~Hz}) \quad$ (b) The 2D space CCF $\rho\left(\delta_{T}, \delta_{R}\right)$ of the simulation model for isotropic scattering (EMED, $N=20$, $f_{\max }=91 \mathrm{~Hz}$ )

Figure $5(a)$ shows the time ACF $\rho_{T}\left(\delta_{T}, \delta_{R}\right)$ of reference and $\hat{\rho}_{T}\left(\delta_{T}, \delta_{R}\right)$ of simulation models; and Figure $5(b)$ shows the 2D space CCF $\rho^{{ }_{T}}\left(\delta_{T}, \delta_{R}\right)$ of stochastic simulation model, for the one ring channel model under isotropic scattering conditions on increasing the number of scatterers to 40 . The simulation parameters used are $N=40, \beta_{B S}=90, \beta_{M S}=90$, $\alpha_{\text {max }}^{\top}=2, f_{\max }=91 \mathrm{~Hz}, k=0, m_{\alpha}=0, \alpha_{v}=180, p=2$ and the parametric computation method used is EMED. Figure 5(a) shows that the time ACF inconsistencies are increased for $T>T_{\max }$ on increasing the number of scatterers. It can be observed from Figure 5(b) that the space CCF decreases by increasing the number of scatterers. However, the decay of the $2 \mathrm{D}$ space CCF $\rho_{11,22}\left(\delta_{T}, \delta_{R}\right)$ is faster along the $\delta_{R}$ direction. 

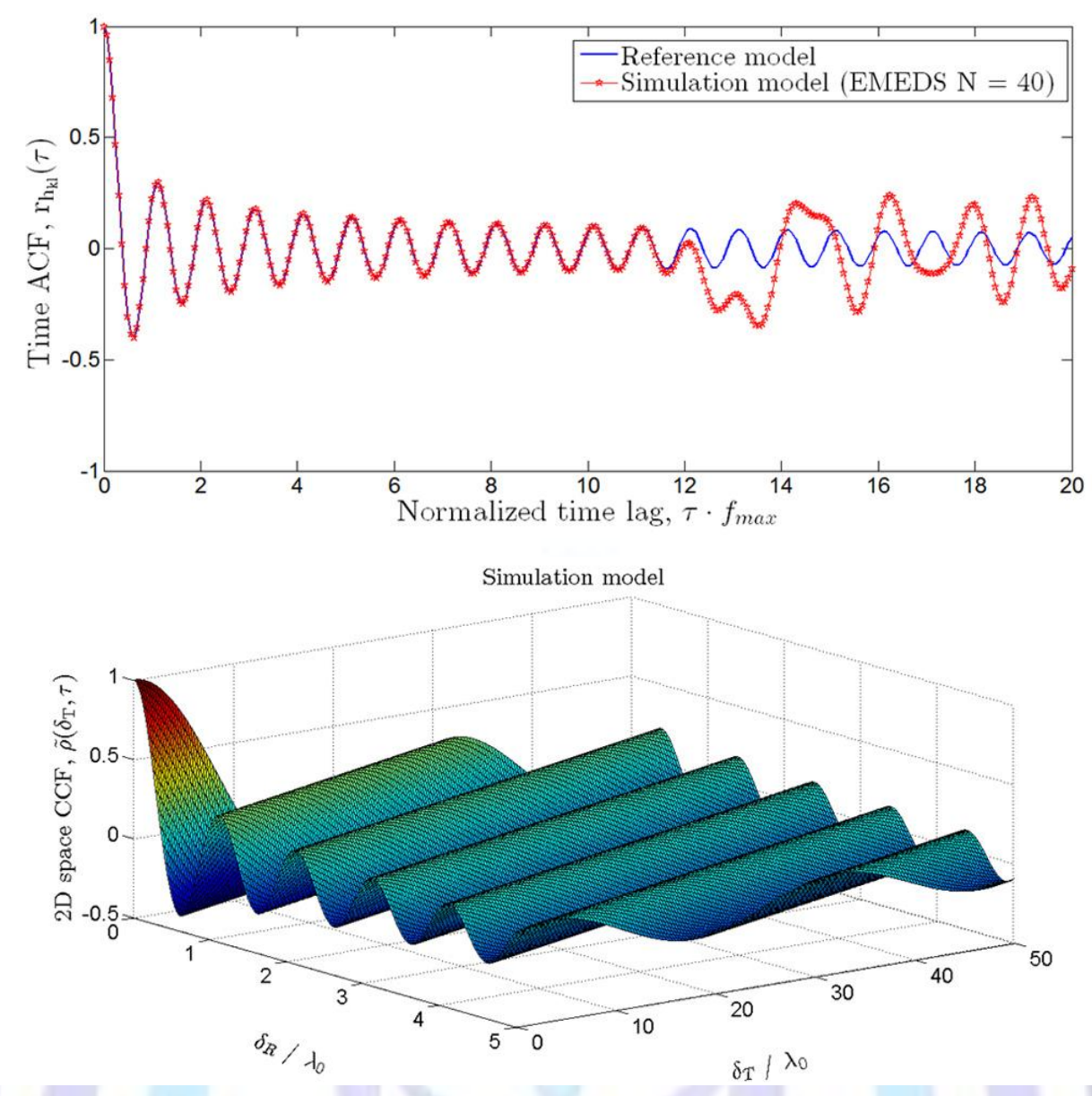

Figure 5: (a) Time ACFs of reference model and simulation model for isotropic scattering (EMED, $N=40, f_{\max }=$ $91 \mathrm{~Hz}$ ) (b) The 2D space CCF $\rho\left(\delta_{T}, \delta_{R}\right)$ of the simulation model for isotropic scattering $\left(E M E D, N=40, f_{\max }=\right.$ 91Hz)

Figures 6(a) and 6(b) illustrate the Time-ACF and 2D space-CCF of the simulation model under isotropic scattering conditions, respectively; for one ring channel model under isotropic scattering conditions. The Doppler frequency is increased here $f_{\max }=200 \mathrm{~Hz}$ and other simulation parameters used are $N=20, \beta_{B S}=90, \beta_{M S}=90, \alpha_{\text {max }}^{\top}=2, f_{\max }=$ $200 \mathrm{~Hz}, k=0, m_{\alpha}=0, \alpha_{v}=180, p=2$. The parametric computation method used is EMED. The results show that the time ACF and 2D space CCF for Doppler frequency $f_{\max }=200 \mathrm{~Hz}$ are same as for Doppler frequency $91 \mathrm{~Hz}$ (Figure $4(\mathrm{a})$ and 4(b)). Moreover, from Figure 6(b), it can be seen that the space CCF is highly correlated over a large range of antenna element spacing's $\delta_{\mathrm{T}}$ and $\delta_{\mathrm{R}}$. This can be concluded from the fact that even for large antenna element spacing's, for example, $\delta_{T}=\delta_{R}=3 \lambda$, the absolute value of the $2 D$ space CCF $\left|\rho_{11,22}\left(\delta_{T}, \delta_{R}\right)\right|$ equals approximately one half of its maximum value.

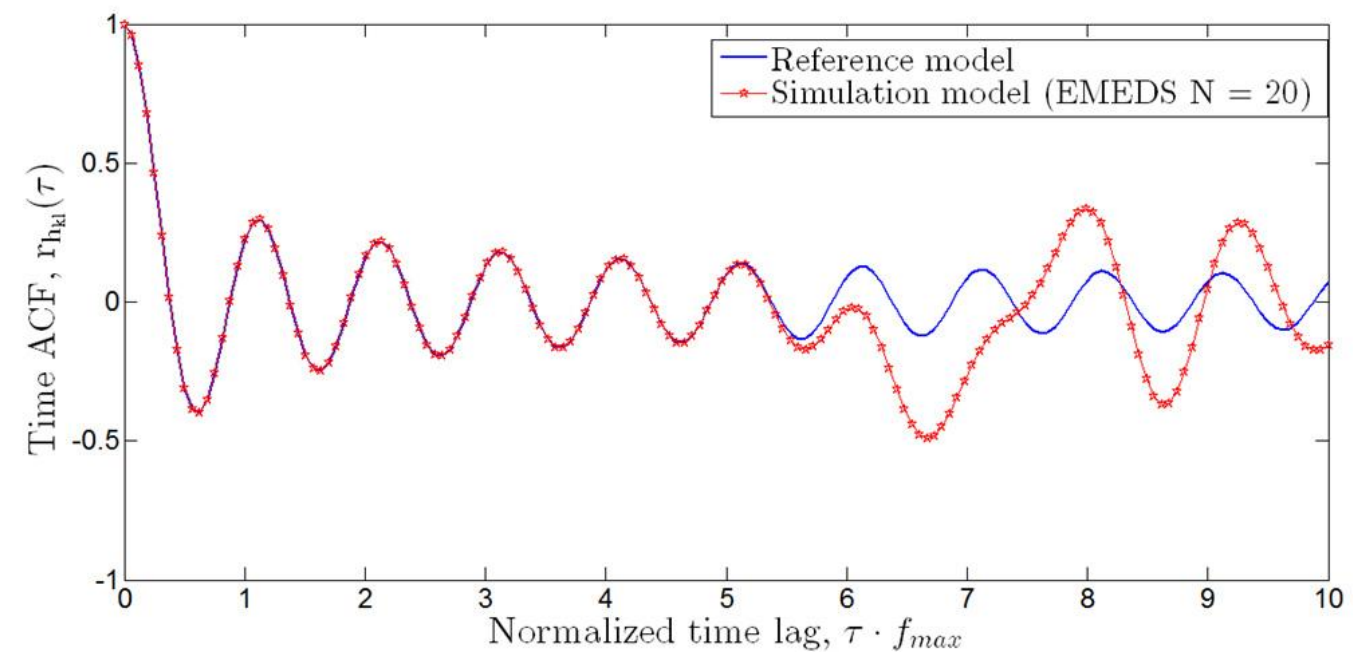




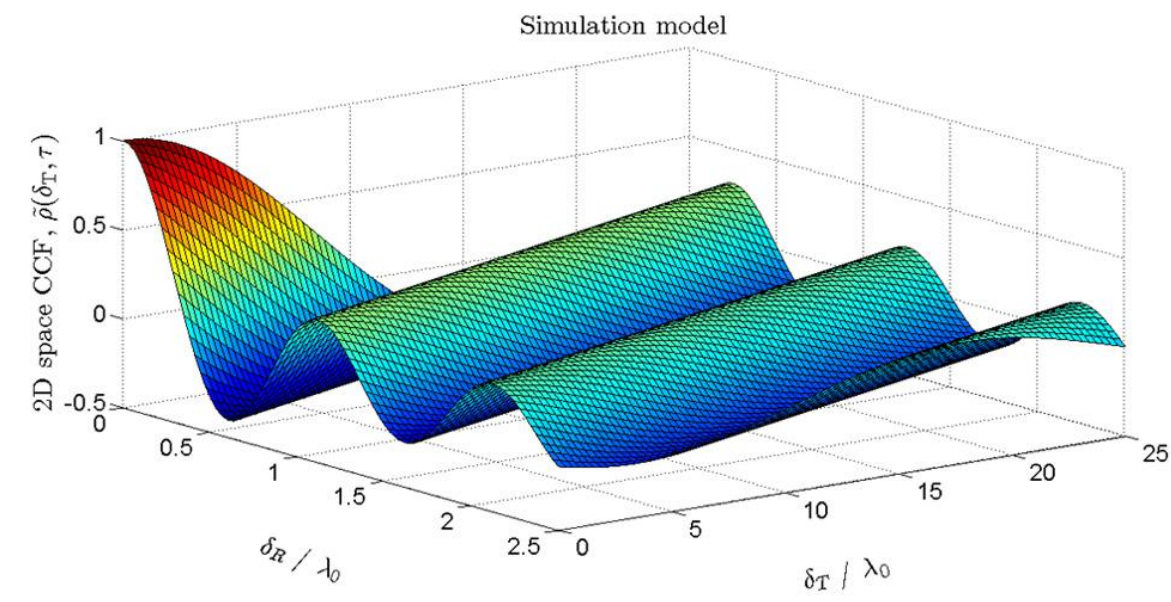

Figure 6: (a) Time ACFs of reference model and simulation model for isotropic scattering (EMED, $N=20, f_{\max }=$ $200 \mathrm{~Hz}$ ) (b) The 2D space CCF $\rho\left(\delta_{T}, \delta_{R}\right)$ of the simulation model for isotropic scattering $\left(E M E D, N=20, f_{\max }=\right.$ 200Hz)

For Non-isotropic Scattering, a high-performance parameter computation method LPNM is used for computing the AOD (angle of departure) as well as AOA (angle of arrival), since the EMEDS cannot be used to determine the model parameters $\alpha_{n}^{R}$ for non-isotropic scattering. Figure $7(\mathrm{a})$ shows the time $A C F \rho_{\mathrm{T}}\left(\delta_{\mathrm{T}}, \delta_{\mathrm{R}}\right)$ of reference and $\rho^{\wedge}{ }_{\mathrm{T}}\left(\delta_{\mathrm{T}}, \delta_{\mathrm{R}}\right)$ simulation models; and Figure $7(\mathrm{~b})$ shows the 2D space CCF $\rho^{\wedge} \mathrm{T}\left(\delta_{\mathrm{T}}, \delta_{\mathrm{R}}\right)$ of stochastic simulation model, for one ring channel model under isotropic scattering conditions. The Lp-norm (LPNM) method $(\kappa=10)$ is applied here optimizes the simulation model parameters by using a finite number of scatterers. The simulation parameters used are $N=20, \beta_{B S}=90$ $\beta_{M S}=90, \alpha_{\max }^{\top}=2, f_{\max }=91 \mathrm{~Hz}, K=10, m_{\alpha}=0, \alpha_{v}=180, p=2$. From Figure $7(a)$, it can be observed that the approximation $r h_{k l}(\tau) \approx \tilde{r} h_{k l}(T)$ is excellent over the interval from 0 to $\tau_{\max }$. The approximation error outside the interval [0, $\pi$ max $]$ is not controlled by the $L p$-norm, $E_{1}^{(p 1)}$. This explains the discrepancy between $r h_{k l}(T)$ and $\tilde{r} h_{k l}(T)$ if $T>I_{\max }$.

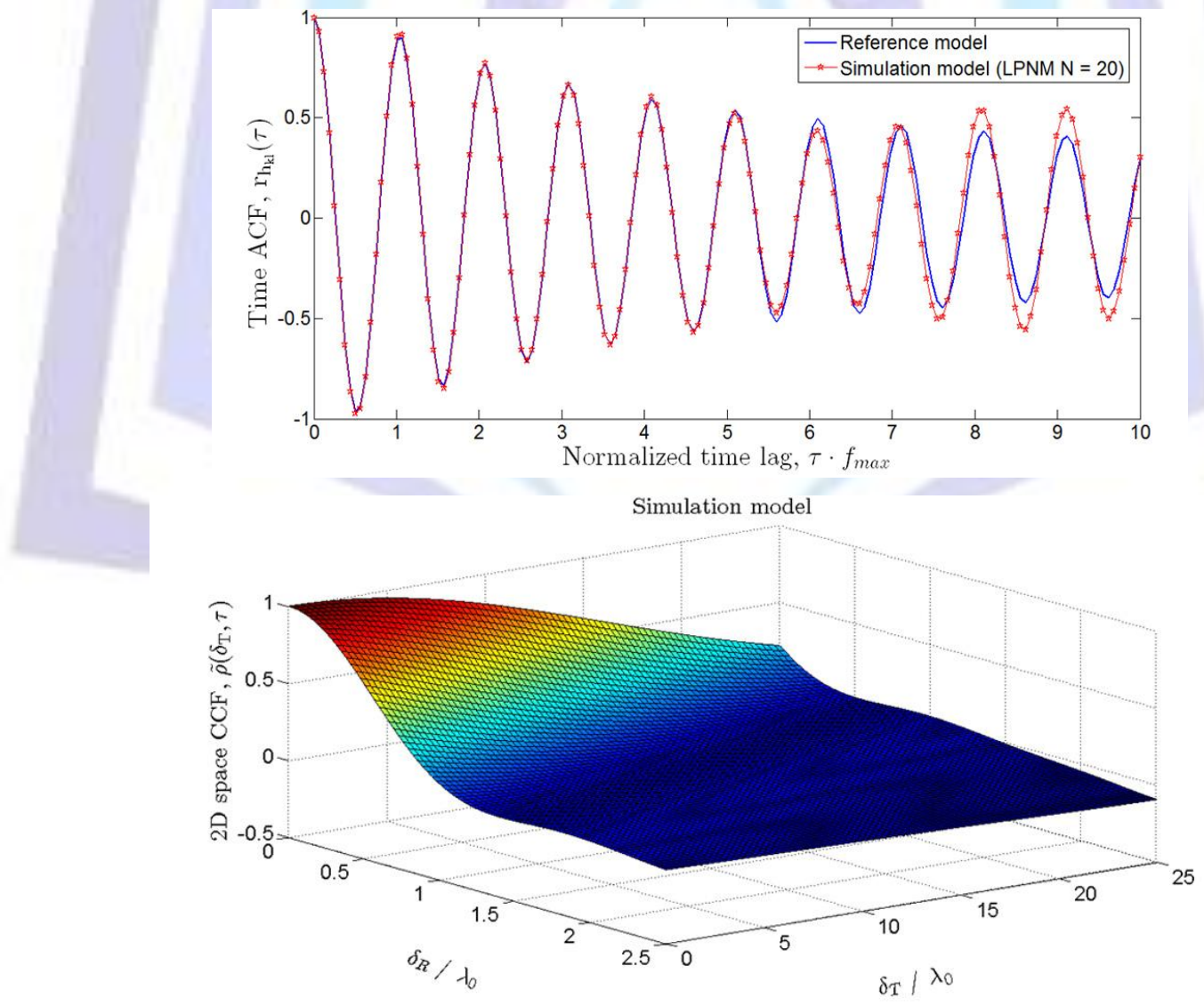

Figure 7: (a) Time ACFs of reference model and simulation model for non-isotropic scattering $\left(L P N M, N=20, f_{\max }=\right.$ $91 \mathrm{~Hz}$ ) (b) The 2D space CCF $\rho\left(\delta_{T}, \delta_{R}\right)$ of the simulation model for non-isotropic scattering $\left(\mathrm{LPNM}, \mathrm{N}=20, \mathrm{f}_{\max }=\right.$ 91Hz)

Figure 8(a) and 8(b) provides information about the time ACF and 2D space CCF parameters for the simulation model when the AODs are non-uniformly distributed on the rings around the MS. The LPNM method for parameter computation 
is used and the number of scatterers are increased $(\mathrm{N}=40)$. The simulation parameters used are $N=40, \beta_{B S}=90, \beta_{M S}=$ $90, \alpha_{\text {max }}^{\top}=2, f_{\max }=91 \mathrm{~Hz}, k=10, m_{\alpha}=0, \alpha_{v}=180$ and $p=2$. From Figure 8(a), it can be observed that the Time-ACF decreases as the time lags increase in isotropic scattering environments.
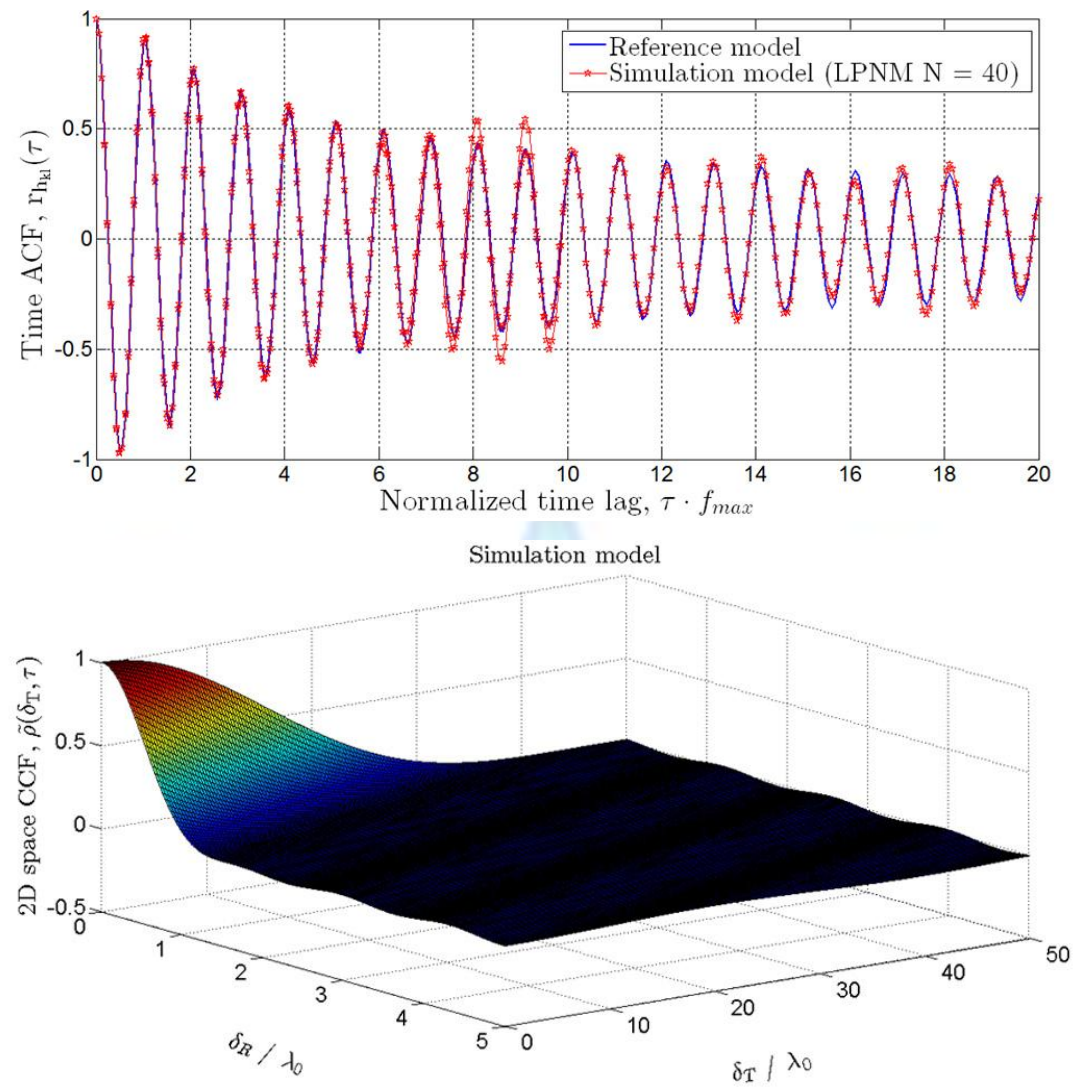

Figure 8: (a) Time ACFs of reference model and simulation model for non-isotropic scattering (LPNM, $N=40, f_{\max }=$ $91 \mathrm{~Hz}$ ) (b) The 2D space CCF $\rho\left(\delta_{T}, \delta_{R}\right)$ of the simulation model for non-isotropic scattering (LPNM, $N=40, \mathrm{f}_{\max }=$ 91Hz)

Figure $9(\mathrm{a})$ shows the time ACF $\rho_{\mathrm{T}}\left(\delta_{\mathrm{T}}, \delta_{\mathrm{R}}\right)$ of reference and $\rho_{\mathrm{T}}\left(\delta_{\mathrm{T}}, \delta_{\mathrm{R}}\right)$ simulation models; and Figure $9(\mathrm{~b})$ shows the space CCF $\rho^{\wedge}\left(\delta_{T}, \delta_{R}\right)$ of stochastic simulation model, for one ring channel model under non-isotropic scattering conditions and using LPNM method for parameter computation. The Doppler frequency is increased to $200 \mathrm{~Hz}$. The simulation parameters used are $\mathrm{N}=20, \beta_{\mathrm{BS}}=90, \beta_{\mathrm{MS}}=90, \alpha_{\max }^{\top}=2, \mathrm{f}_{\max }=200 \mathrm{~Hz}, k=10, \mathrm{~m}_{\alpha}=0, \alpha_{v}=180, p=2$. The results with Doppler frequency $200 \mathrm{~Hz}$ are same as that of $91 \mathrm{~Hz}$ (Figure $7(\mathrm{a})$ and $7(\mathrm{~b})$ ). A performance investigation of the LPNM revealed that this method gives best fitting parameter values for non-isotropic scatter model.

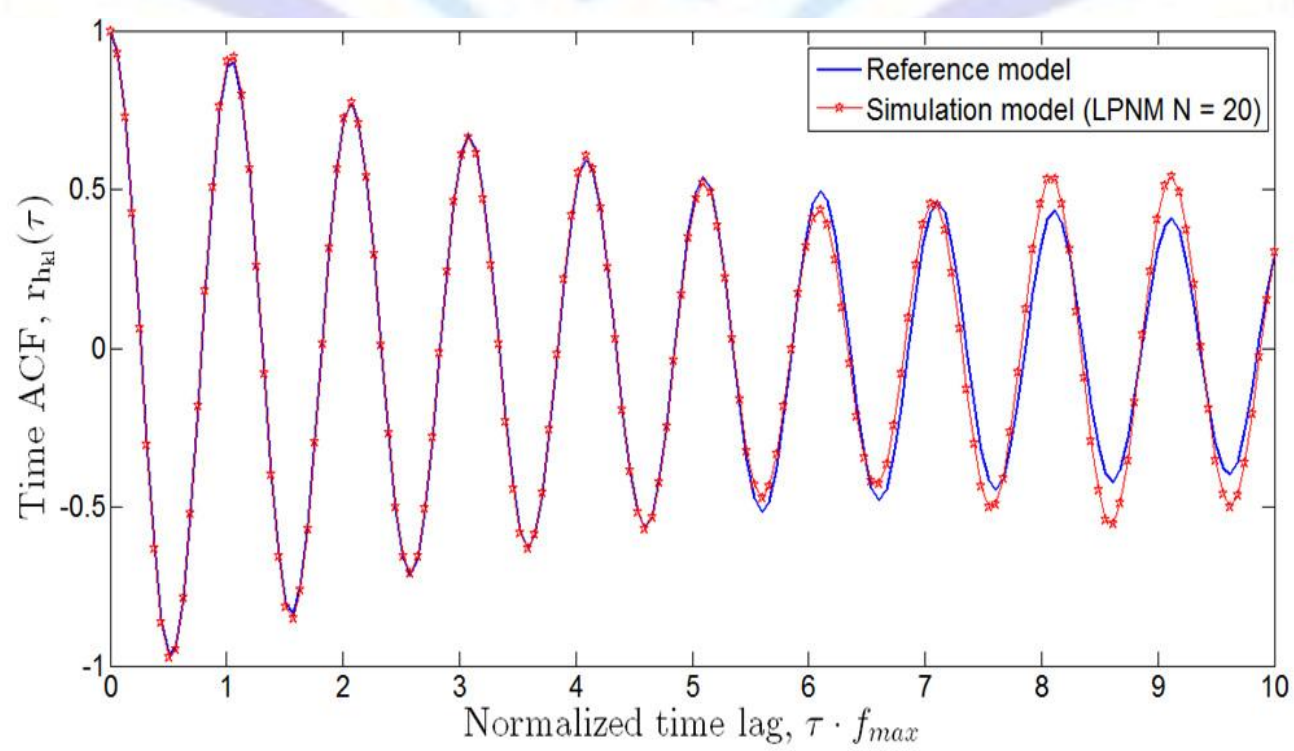




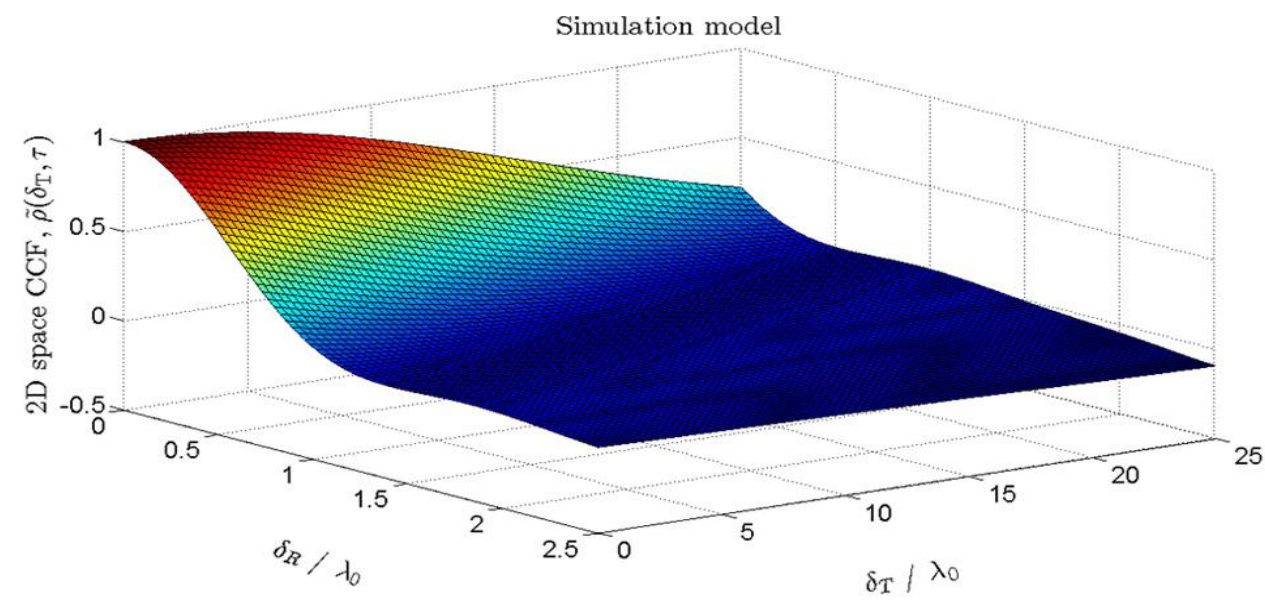

Figure 9: (a) Time ACFs of reference model and simulation model for non-isotropic scattering $\left(L P N M, N=20, f_{\max }=\right.$ $200 \mathrm{~Hz}$ ) (b) The 2D space CCF $\rho\left(\delta_{T}, \delta_{R}\right)$ of the simulation model for non-isotropic scattering $\left(L P N M, N=20, f_{\max }=\right.$ 200Hz)

Now, Figure $10(a)$ and 10 (b) shows the simulation of the MIMO channel capacity for $M_{T}=M_{R}=2$ and $M_{T}=M_{R}=4$ respectively, at $S N R=0 \mathrm{~dB}$. It is demonstrated here that, how the channel simulations could be used to analyze the statistics of the capacity of MIMO channel models. The ecdf (Empirical Cumulative Distribution Function) is used to evaluate the channel capacity distribution. From Figure 10(a), it can be seen that a capacity of $2 \mathrm{bps} / \mathrm{Hz}$ (for example) can be acquired with the probability of 0.8 . Also, a capacity of $1.35 \mathrm{bps} / \mathrm{Hz}$ is equi-probable; means cumulative probability is 0.5 . Figure $10(\mathrm{~b})$ shows that capacity of $2.2 \mathrm{bps} / \mathrm{Hz}$ can be obtained with probability of 0.5 .
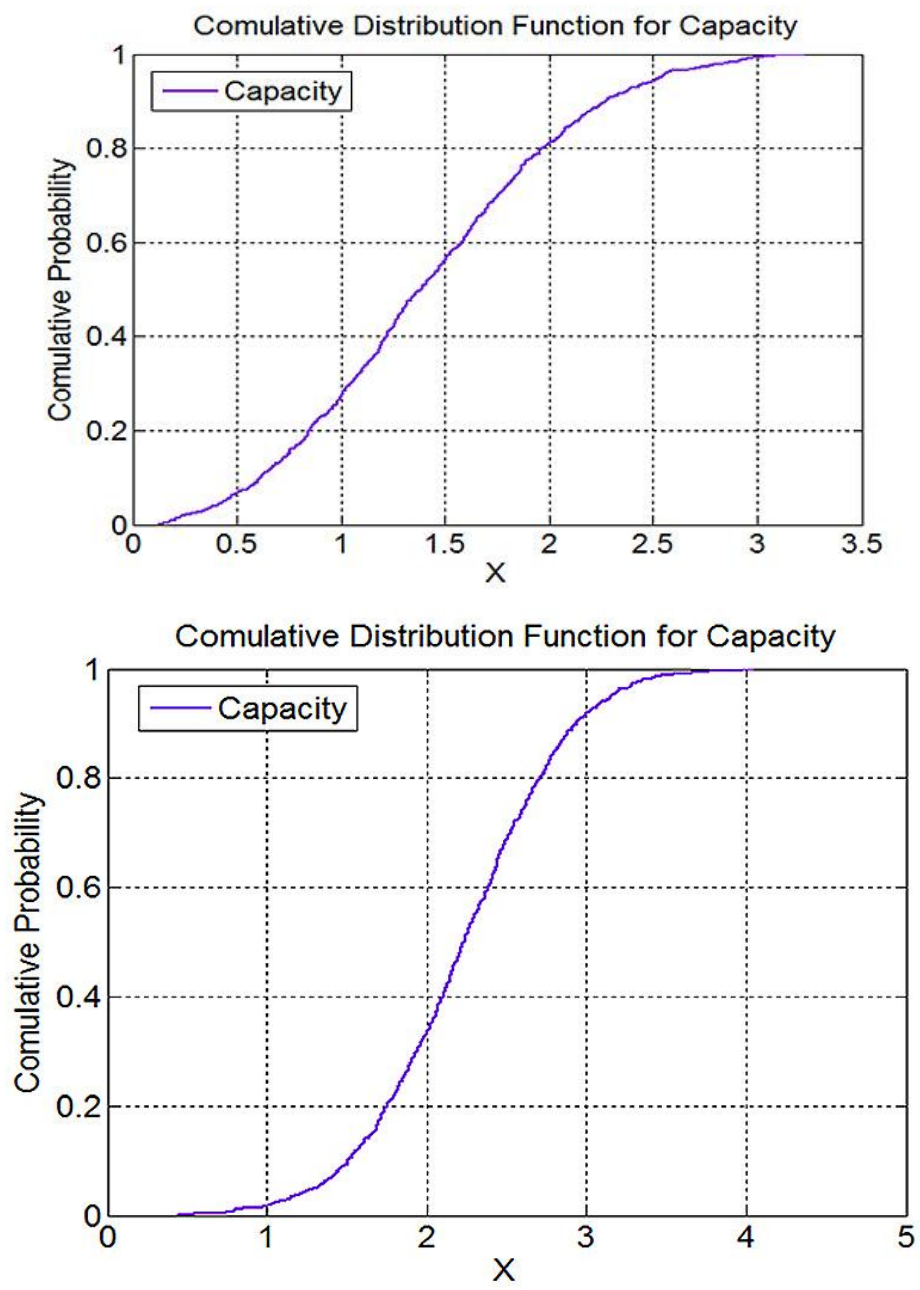

Figure 10: (a) ecdf for channel capacity $C(t)$ of the two ring $2 \times 2$ MIMO channel model $(N=40, S N R=0)$ (b) ecdf for channel capacity $C(t)$ of the two ring $4 \times 4$ MIMO channel model $(\mathrm{N}=40, \mathrm{SNR}=0)$ 
For Two Ring Channel Models, the scatterers are assumed to present at the MS as well as BS. The simulation results are based on the Isotropic Scattering assumption, i.e., $p(\varphi T)=p(\varphi R)=1 /(2 \pi)$. The tilt angles $\beta_{T}$ and $\beta_{R}$ are defined as $\beta_{T}=\beta_{T}$ $=\pi / 2$, and the angle of motion $\alpha_{v}$ is set to $\pi$. The ring radii $R_{T}$ and $R_{R}=10 \mathrm{~m}$; maximum Doppler frequency, $f_{\max }=91 \mathrm{~Hz}$, is assumed, and the wave length $\lambda$ is set to $0.15 \mathrm{~m}$. The parameters $\varphi(m) T$ and $\varphi(n) R$ of the simulation model are computed by using the proposed EMEDS with $M=20$ (number of scatterers at the BS) and $N=40$ (number of scatterers at the MS). The other simulation parameters are; $M=40, \alpha^{\top}{ }_{v}=180, \beta_{T}=90, k=0$ and $p=2$. The time ACF is same for reference and simulation model as shown in Figure 11(a). Also from Figure 11(b), it can be seen that the space CCFs are highly correlated over a large range of antenna element spacing's.
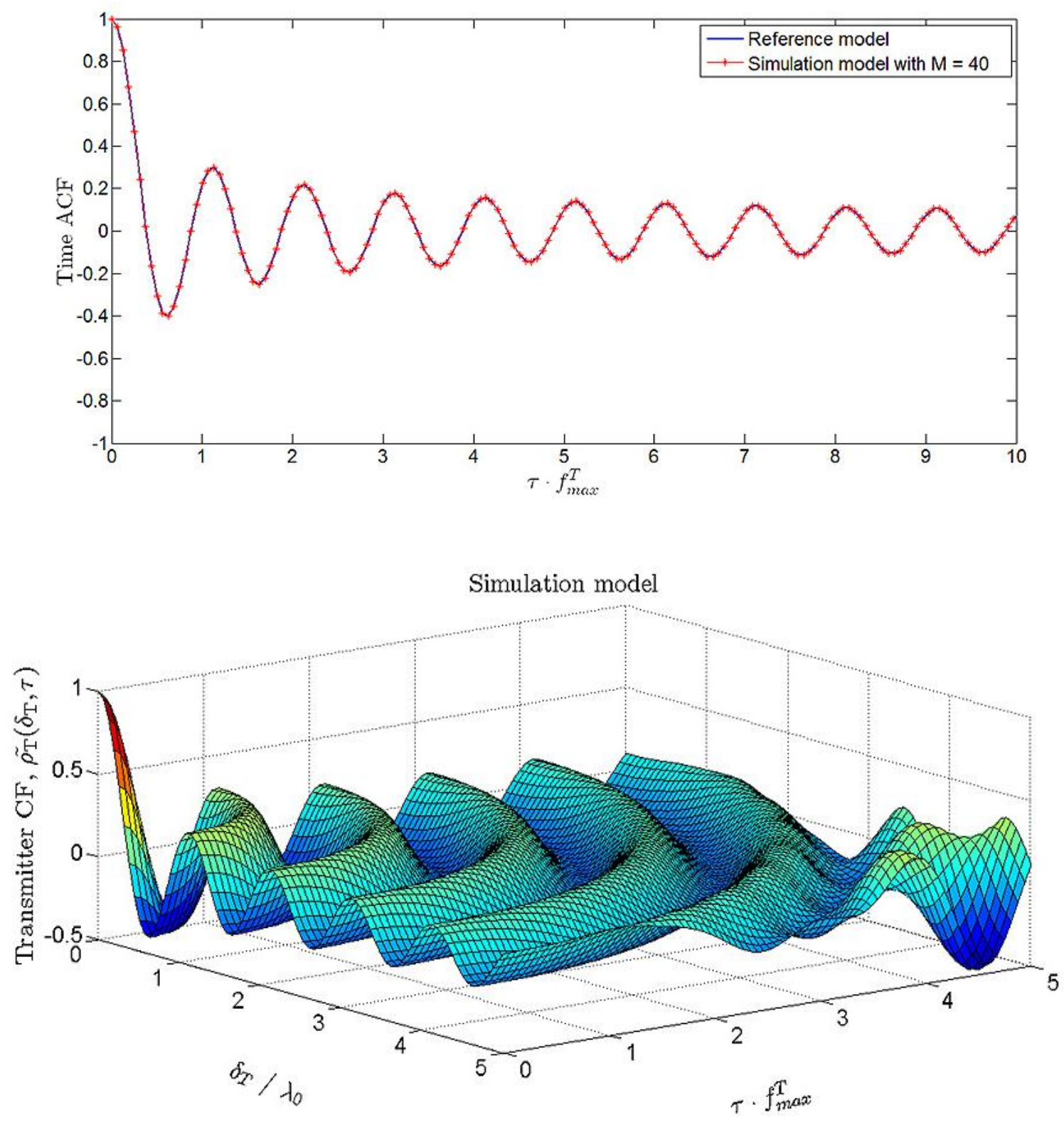

Figure 11: (a) Time ACFs of reference model and simulation model for isotropic scattering (EMED, $M=40, f_{\max }=$ $91 \mathrm{~Hz})$ (b) The 2D space CCF $\rho\left(\delta_{T}, \delta_{R}\right)$ of the simulation model for isotropic scattering $\left(E M E D, M=40, f_{\max }=\right.$ $91 \mathrm{~Hz})$

Figure 12(a) and 12(b) illustrates the Time-ACF and space-CCF of the simulation model under isotropic scattering conditions, respectively, for two ring channel model. The method used here to optimize the simulation model parameters using a finite number of scatterers, is $\operatorname{EMED}(k=0)$. The simulation parameters used are; $M=60, \alpha_{v}^{\top}=180, f^{\top}{ }_{\max }=91 \mathrm{~Hz}$, $\beta_{T}=90, k=0$ and $p=2$. The number of scatterers is further increased. Form Figure 12(a), it can be observed that the 2D space CCF decreases as the antenna inter-element spacing is increased. Figure 12(b) shows that under isotropic scattering conditions, the close mapping of the models is obtained, with the increase in number of scatters $N$. 

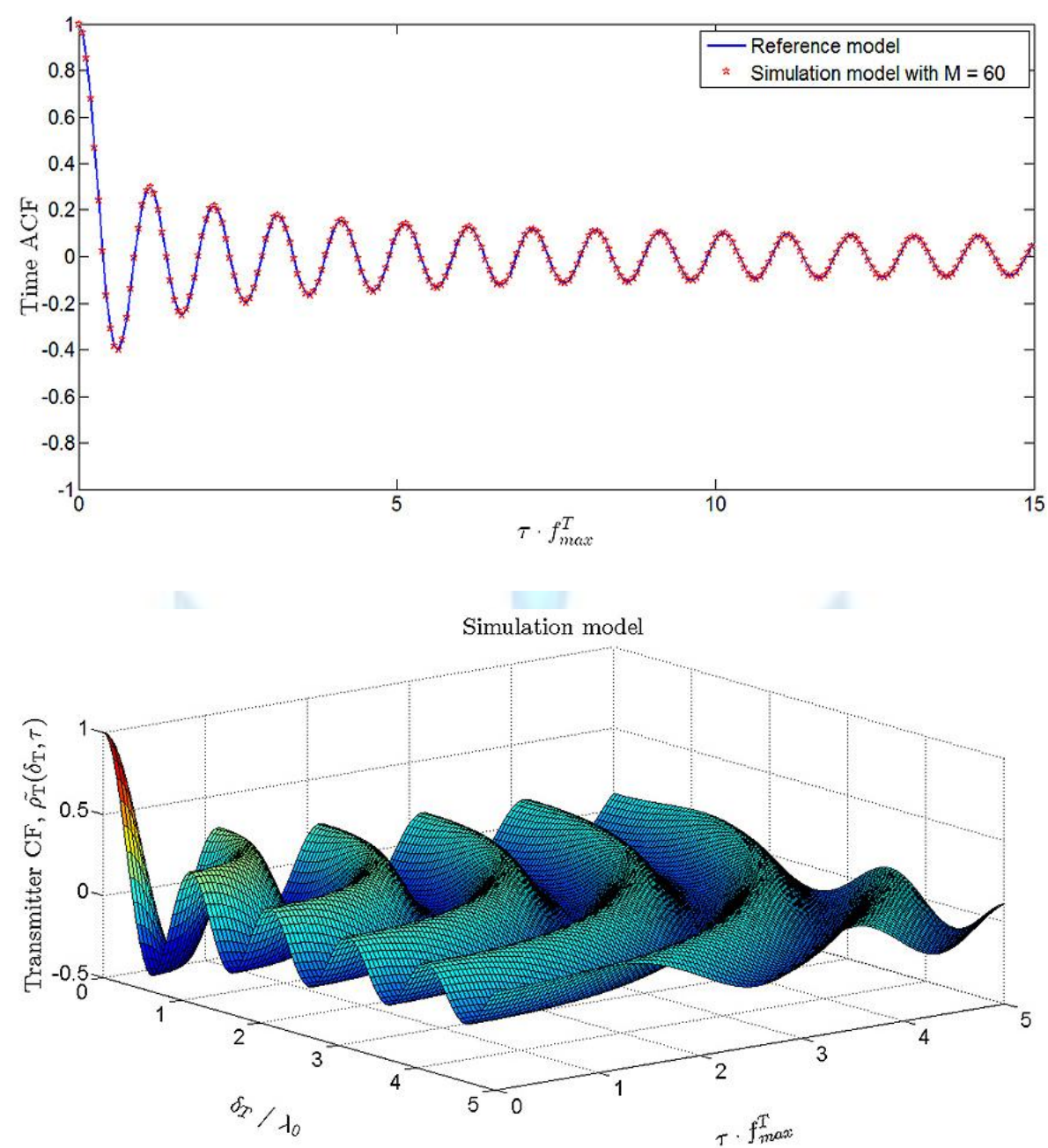

Figure 12: (a) Time ACFs of reference model and simulation model for isotropic scattering (EMED, $M=60, f_{\max }=$ $91 \mathrm{~Hz}$ ) (b) The 2D space CCF $\rho\left(\delta_{T}, \delta_{R}\right)$ of the simulation model for isotropic scattering (EMED, $M=60, f_{\max }=$ 91 Hz)

For non-isotropic scattering conditions, the LPNM method is applied to optimize the simulation model parameters by using a finite number of scatterers. For the simulation model, $M=40$ scatterers at the MS are assumed. Figures 13(a) and 13(b) illustrate the Time-ACF $\left(\rho_{\mathrm{T}}\left(\delta_{\mathrm{T}}, \delta_{\mathrm{R}}\right)\right.$ and $\left.\rho^{\wedge} \mathrm{T}\left(\delta_{\mathrm{T}}, \delta_{\mathrm{R}}\right)\right)$ and $2 \mathrm{D}$ space-CCF $\rho_{\mathrm{T}}{ }_{\mathrm{T}}\left(\delta_{\mathrm{T}}, \delta_{\mathrm{R}}\right)$ of the simulation model under nonisotropic scattering conditions, respectively. The simulation parameters used are; $M=40, \alpha_{v}^{\top}=180, f^{\top}{ }_{\max }=91 \mathrm{~Hz}, \beta_{\mathrm{T}}=90$, $\kappa=40$ and $p=2$. Here the Von Mises density is used to compute AOA with parameter $k$ and $m_{\alpha}$. A performance investigation of the LPNM revealed that this method outperforms the EMEDS only slightly in case of isotropic scattering.

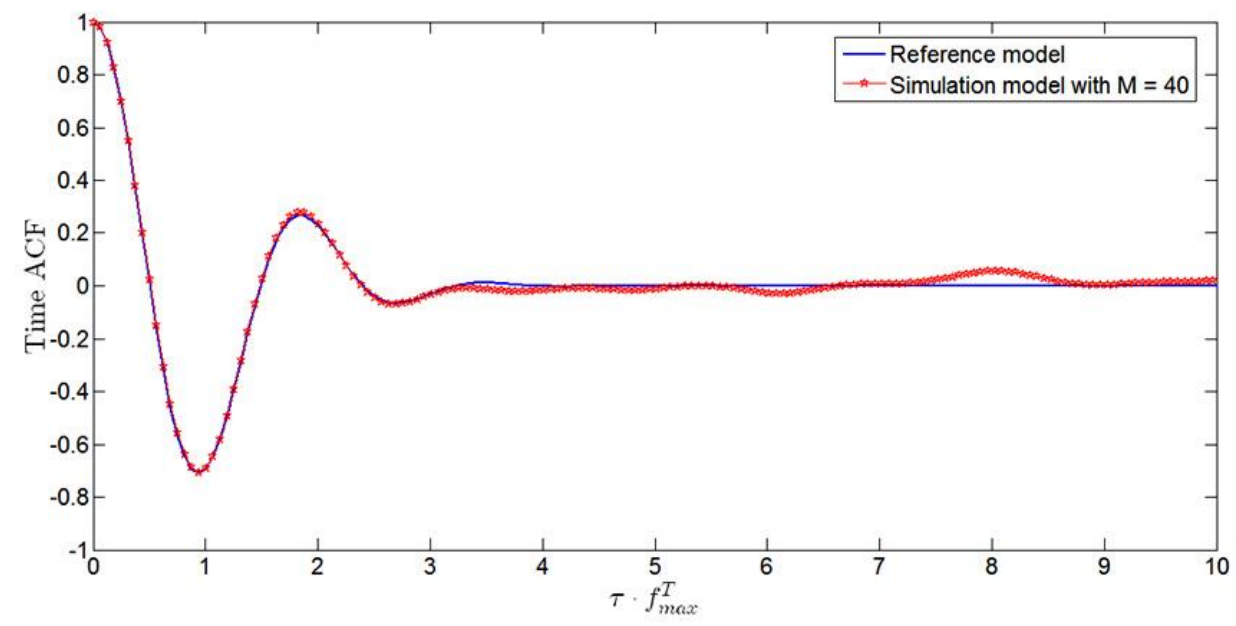




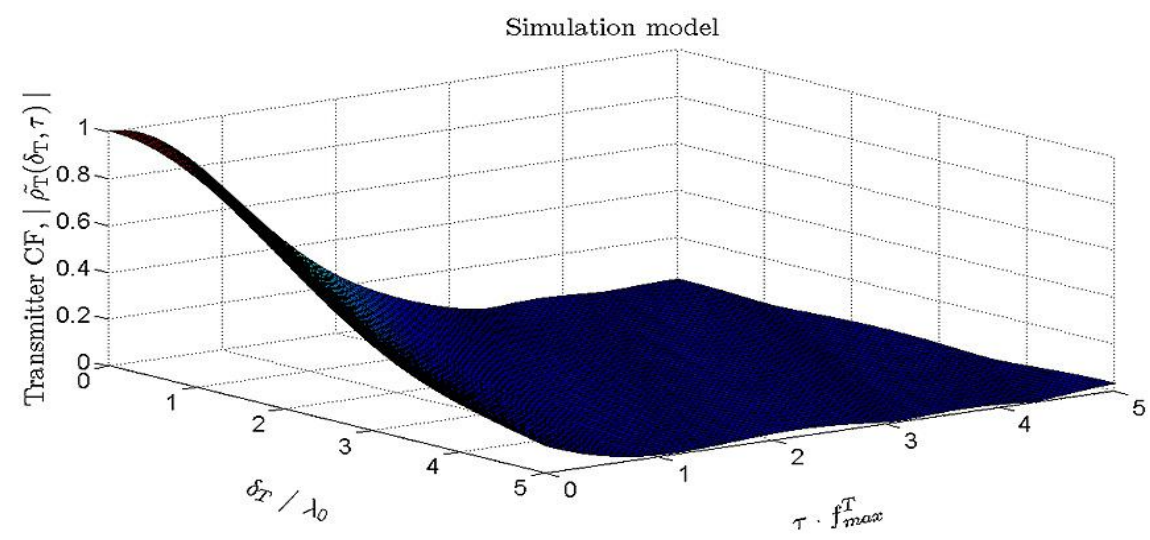

Figure 13: (a) Time ACFs of reference model and simulation model for non-isotropic scattering (LPNM, M=40, $f_{\max }$ $=91 \mathrm{~Hz})$ (b) The 2D space CCF $\rho\left(\delta_{T}, \delta_{R}\right)$ of the simulation model for non-isotropic scattering (LPNM, M=40, $f_{\max }=91 \mathrm{~Hz}$ )

Figure 14(a) and 14(b) demonstrate that, how the channel simulations could be used to analyze the statistics of the capacity of $2 \times 2$ and $4 \times 4$ MIMO channel models, respectively. The ecdf is used to evaluate the channel capacity distribution. The simulation parameters used are $\mathrm{Mt}=\mathrm{Mr}=2$ and 4 respectively, $\mathrm{SNR}=0, \mathrm{M}=20$ and $\mathrm{N}=40$. From Figure 26 , it can be seen that, a capacity of $20 \mathrm{bps} / \mathrm{Hz}$ can be obtained with equal probability, i.e. 0.5 . Besides, from Figure 27 , it can be found that a capacity of $26.5 \mathrm{bps} / \mathrm{Hz}$ can be obtained with probability of 0.5 .
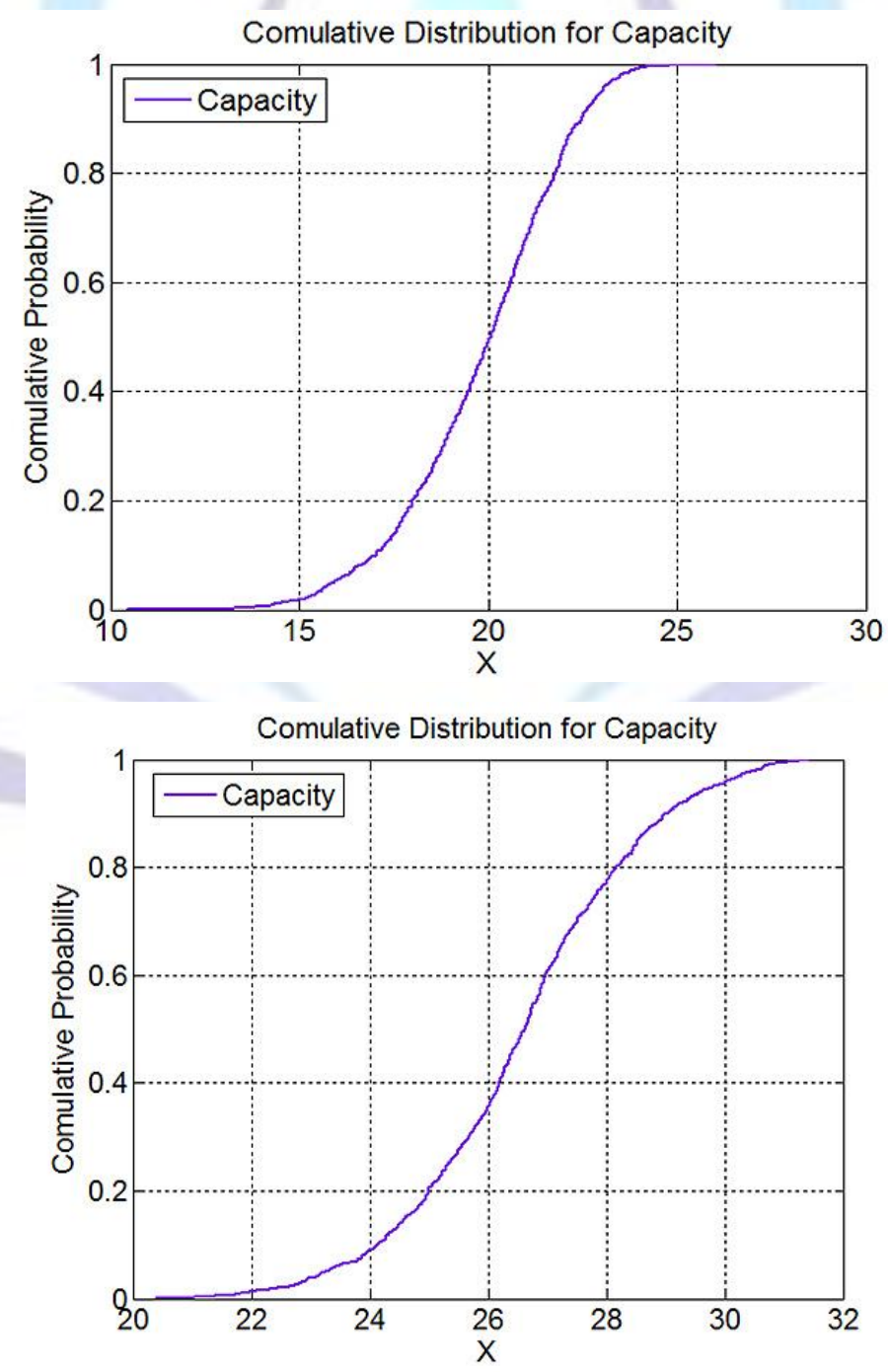

Figure 14: (a )ecdf for channel capacity $C(t)$ of the two ring $2 \times 2$ MIMO channel model $(N=40$, SNR=0) (b) ecdf for channel capacity $C(t)$ of the two ring $4 \times 4$ MIMO channel model $(N=40, S N R=0)$ 
By analyzing these figures, the simulation results for different parameter values coming from the EMEDS method for isotropic scattering and the LPNM method for non-isotropic are found. For each parameter computation method, the ACF and CCF of the simulation model are acquired. By increasing the number of scatterers, the Space CCF $\rho_{11,22}\left(\delta_{T}, \delta_{R}\right)$ decreases quickly along the $\delta_{R}$ direction. However, the Time ACF and Space CCF remain the same by increasing the Doppler requency. On comparing the CCF of above figures, it shows that best performance is given in simulation results for non-isotropic scattering model, as compared to the isotropic scattering model. Reason behind this better performance is LPNM methods given best fitting parameter values for non-isotropic scatter model. The LPNM can be applied for minimizing the error function of the one-ring model parameters and the two-ring model parameters. Thus, LPNM is equally beneficial for the computation of the channel model parameters under non-isotropic scattering conditions. Under isotropic scattering conditions, a very good fitting is obtained until $\delta R / \lambda=N / 4$ and $\delta_{T} / \lambda=N / 4$. With the increase of number of scatters $N$, the close mapping of the models is obtained. Therefore, an efficient adaptive modulation techniques are required.

\section{CONCLUSION}

A profound knowledge of mobile fading channels is required for the development, evaluation, and assessment at practical conditions of present and future wireless communication systems. Hence, the modeling, analysis, and simulation of MIMO fading channels are important segment for the initiation of mobile communications. This paper deals with the performance analysis and simulation of MIMO fading channels for mobile communication systems using one ring and two ring models. The Temporal and Spatial correlation behavioral characteristics of MIMO fading channels are investigated using One-Ring and Two-Ring models. For one-ring model, it is assumed that only the transmitter, which is the MS, is surrounded by an infinite number of local scatterers. Whereas, for the two-ring models, it is assumed that the scattering objects are located near both the transmitter (BS) and the receiver (MS). A general procedure is presented for the design of stochastic simulation models for frequency- nonselective MIMO fading channels based on the geometrical one ring and two-ring scattering models under different channel conditions.

Two methods are used for computing the parameters of the simulation model; i.e. the extended MEDS (EMEDS) for isotropic scattering and a variant of the Lp-norm (LPNM) method for non-isotropic scattering. A performance investigation of the LPNM revealed that this method outperforms the EMEDS slightly in case of isotropic scattering. The system performance decreases if the spatial correlation is increased. With increasing the number of scatterers, the performance of channel models degrades, thus efficient adaptive modulation techniques are required for better performance. Besides, the MIMO channel simulations enable an exact analysis of the channel capacity by simulation. The proposed procedure for the design of MIMO channels provides a fundamental framework for the test, optimization, design, and analysis of multielement antenna communication systems.

\section{References}

[1] Patzold, M., Youssef, N., and Carlos, A. G., 2012. Modeling and Simulation of Mobile Radio Channels. Modelling and Simulation in Engineering, Hindawi Publishing Corporation, Article ID 160297, doi:10.1155/2012/160297

[2] Yu, K., and Ottersten, B., 2002. Models for MIMO Propagation Channels, A Review. Wiley Journal on Wireless Communications and Mobile Computing Special Issue on Adaptive Antennas and MIMO Systems

[3] Shiu, D.S., Foschini, G. J., Gans, M. J., and Kahn, J. M., 2000. Fading Correlation and Its Effect on the Capacity of Multielement Antenna Systems, IEEE Transactions on Communications. Vol. 48, No. 3

[4] Oestges, C., and Clerckx, B., 2007. MIMO Wireless Communications- From real-world propagation to space time code design. Publisher: Academic Press, Elsevier Ltd., ISBN 13: 9-78-0-12-372535-6

[5] Cheng, X., Laurenson, D. I., and Salous, S., 2009. An Adaptive Geometry-Based Stochastic Model for Non-Isotropic MIMO Mobile-to-Mobile Channels. IEEE Transactions on Wireless Communications

[6] Patzold, M., 2012. Mobile Radio Channels, Second Edition, Publisher: John Wiley \& Sons, Ltd., ISBN: 978-1-11997412-3

[7] Tsoulos, G., 2006. MIMO System Technology for Wireless Communications, Publisher- CRC Press, Taylor \& Francis Group. ISBN-13: 978-0-8493-4190-8

[8] Parizi, F. Z., Mehrjoo, M., and Shokouh, J. A., 2013. A Survey of Geometrically-Based MIMO Propagation Channel Models. Technical Journal of Engineering and Applied Sciences. ISSN 2051-0853

[9] Avazov, N., and Patzold, M., 2012. Design of Wideband MIMO Car-to-Car Channel Models Based on the Geometrical Street Scattering Model. Modelling and Simulation in Engineering, Hindawi Publishing Corporation, Article ID 264213 , doi:10.1155/2012/264213

[10] Wang, S., Abdi, A., Wallace, J. W., and Jensen, M. A., 2005. Time-Varying MIMO Channels: Parametric Statistical Modeling and Experimental Results. IEEE International Workshop on Signal Processing Advances for Wireless Communications, New York City

[11] Talha, B., and Patzold, M., 2011. A Geometrical Three-Ring-Based Model for MIMO Mobile-to-Mobile Fading Channels in Cooperative Networks. EURASIP Journal on Advances in Signal Processing. Hindawi Publishing Corporation. Article ID 892871, doi:10.1155/2011/892871 
[12] Cheng, X., Wang, C. X., and Laurenson, D. I., 2009. Multiple-Ring Based Modeling and Simulation of Wideband Space-Time-Frequency MIMO Channels. IEEE International Conference on Communications.

[13] Patzold, M., 2004. Modeling and Simulation of MIMO Channels. BEATS/CUBAN Workshop

[14] Bakhshi, G., Saadat, R., and Shahtalebi, K., 2012. Modeling and Simulation of MIMO Mobile-to-Mobile Wireless Fading Channels. International Journal of Antennas and Propagation, Hindawi Publishing Corporation. Article ID 846153, doi:10.1155/2012/846153

\section{Author' biography}

Rachna Mahey B.Tech., M.Tech (pursuing) is student at Electronics and Communications Department, Guru Nanak Dev University, Regional Campus, Jalandhar, India. Her research areas include Performance Characterization of Fading Channels and MIMO wireless systems.

Dr. Jyoteesh Malhotra B.Eng., M.Tech, Ph.D. is involved in teaching and research at Electronics and Communications Department, Guru Nanak Dev University, Regional Campus, Jalandhar, India. His research areas of interest include Statistical modeling of Fading Channels and Fading mitgitaion techniques in Wireless Communication. Dr. Malhotra has more than 100 research publications and authored 02 books. He is a life member of I.S.T.E. and editorial board of many International Journals of repute. 